\title{
Differentiation of Mouse Embryonic Stem Cells to Neuronal Cells Using Hanging Droplets and Retinoic Acid \\ Jeroen Witteveldt* and Sara Macias
}

\author{
Institute of Immunology and Infection Research, School of Biological Sciences, University of Edinburgh, \\ Edinburgh, EH9 3FL, UK \\ *For correspondence: Jeroen.witteveldt@ed.ac.uk
}

\begin{abstract}
[Abstract] Controlled differentiation of embryonic stem cells is an essential tool in stem cell research. In this protocol, we describe a simple differentiation protocol involving the induction of embryoid body formation in mouse embryonic stem cells (mESC) using hanging droplets, followed by differentiation into a neuronal lineage.
\end{abstract}

Keywords: Embryonic stem cells, Embryoid bodies, Retinoic acid, Hanging droplets, Differentiation, Neuronal

[Background] One of the major achievements in embryonic stem cell research has been the ability to direct the differentiation of stem cells into specific developmental lineages and ultimately terminally differentiated cells. To preserve pluripotency of mESCs in in vitro culture, cells are grown in the presence of the Leukemia inhibitory factor (LIF) cytokine, which promotes self-renewal and inhibits differentiation. A common method to direct stem cell differentiation into all three germ layer lineages is to remove LIF from the culture medium and forcing the formation of complex three-dimensional cell aggregates termed embryoid bodies (EBs) (Itskovitz-Eldor et al., 2000). Differentiation in these embryoid bodies is considered to be disorganized, however mESC culture in the absence of LIF and in the presence of certain stimuli can result in more specific cell types, including insulin-producing cells, hematopoietic cells and neuronal cells. Differentiation of ESCs into neural lineage involves the manipulation of Fibroblast Growth Factor and Wnt signaling in the absence of Bone morphogenetic protein signaling activity (Abranches et al., 2009). Common methods involve co-culture of ESCs on stromal feeder cells or conditioned medium or direct manipulation of the essential signaling pathways which can be quite involved and time-consuming. A more straightforward method is by incubating ESCs with the natural metabolite of vitamin A, retinoic acid which acts on nuclear receptors to induce transcription of specific target genes. Retinoic acid has been shown to play a directing role in neural development in early stages of central nervous system development and is considered the most important extrinsic inductive signals for neural differentiation. In this protocol, we describe an efficient method to produce mouse-derived EBs and consequent neuronal differentiation using hanging droplets and retinoic acid (Jones-Villeneuve et al., 1983; Wobus et al., 1991). 


\section{Materials and Reagents}

1. Pipette tips

2. Pipettes

3. Petri dish (145 mm, Greiner Bio-One, catalog number: 639161; $90 \mathrm{~mm}$, SLS select, catalog number: SLS2002)

4. Cell culture dish (100 mm, Corning, catalog number: 430167)

5. Flask ( $25 \mathrm{~cm}^{2}$, Corning, catalog number: $430639 ; 75 \mathrm{~cm}^{2}$, Corning, catalog number: $\left.430641 \mathrm{U}\right)$

6. $15 \mathrm{ml}$ tube (Sarstedt, catalog number: 62547004)

7. $50 \mathrm{ml}$ tube (Sarstedt, catalog number: 62554002)

8. Cryo tube

9. Mouse Embryonic Stem cells (mESC) (v6.5, Novus Biologicals, catalog number: NBP1-41162) Note: Be sure to check local guidelines regarding the use of embryonic stem cells.

10. DMEM (High glucose, pyruvate, Gibco, catalog number: 41966)

11. Dulbecco's phosphate buffered saline (Sigma-Aldrich, catalog number: D8537)

12. MEM Non-Essential amino acids (Gibco, catalog number: 11140035)

13. Trypsin-EDTA (0.25\%, Gibco, catalog number: 25200056$)$

14. 2-Mercaptoethanol (50 mM, Gibco, catalog number: 31350010)

15. Gelatin (0.1\% in Ultrapure water, Millipore, catalog number: ES-006-B)

16. Fetal bovine serum (Gibco, catalog number: 10500064)

17. L-Glutamine (200 mM, Gibco, catalog number: 25030024)

18. Penicillin/Streptomycin (Gibco, catalog number: 15140122)

19. Retinoic acid (Sigma-Aldrich, catalog number: $2625-50 \mathrm{mg}$ )

20. Leukemia inhibitory factor (LIF, Stemcell Technologies, catalog number: 78056)

21. Liquid nitrogen

22. mESC medium with LIF (see Recipes)

23. $\mathrm{mESC}$ medium without LIF (see Recipes)

24. $0.05 \%$ Trypsin (see Recipes)

\section{Equipment}

1. Incubator $\left(37^{\circ} \mathrm{C}, 5 \% \mathrm{CO}_{2}\right.$, Thermo Scientific, model: BB15)

2. Centrifuge (Eppendorf, model: $5810 \mathrm{R}$ )

3. Tissue-culture hood (Nuaire Labgard, Class II)

4. Hemocytometer (Neubauer chamber or equivalent method)

5. Inverted microscope (Olympus, model: CKX41)

6. Non-inverted microscope (Olympus, model: BX43)

7. Water bath 


\section{Procedure}

A. Coating of plates

1. Add enough $0.1 \%$ Gelatin to cover the bottom of a tissue-culture treated flask or plate (for example, $1.5 \mathrm{ml}$ of $0.1 \%$ Gelatin for a T25 flask).

2. Incubate for at least $10 \mathrm{~min}$ at room temperature or $37^{\circ} \mathrm{C}$.

3. Carefully remove the $0.1 \%$ Gelatin and add mouse embryonic stem cell (mESC) medium (see Recipes).

Note: Washing plates is not necessary when carefully removing gelatin.

B. Recovery of cells from liquid nitrogen

1. Prepare suitable plate as described above.

2. Collect cryo tube with mESCs from liquid nitrogen and quickly thaw in a water bath at $37^{\circ} \mathrm{C}$.

3. Slowly add culture medium with LIF to cells in cryo tube and transfer to a $15 \mathrm{ml}$ tube with approximately $10 \mathrm{ml}$ of medium with LIF.

Note: Slowly adding medium to ESCs prevents damage due to sudden changes in osmotic pressure.

4. Pellet cells for $5 \mathrm{~min}$ at $200 \times g$ and remove supernatant

5. Resuspend cells in appropriate amount of medium with LIF and add to gelatin-coated plate.

C. Maintaining and splitting mESCs

1. Remove mESC medium from flask or plate and wash cells with PBS.

Note: See Figure 1 for example of $v 6.5$ cells ready for splitting.

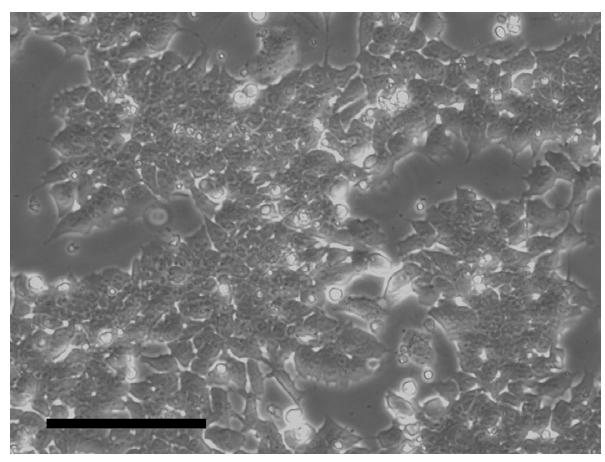

Figure 1. Confluent v6.5 mESCs. Scale bar $=50 \mu \mathrm{m}$.

2. Remove PBS carefully and add enough $0.05 \%$ Trypsin (see Recipes) to cover cells (for example, $1 \mathrm{ml}$ of $0.05 \%$ trypsin in a T25 flask).

3. Incubate at $37^{\circ} \mathrm{C}$. Usually $3-5 \mathrm{~min}$ is enough for all cells to dissociate.

Note: Do not leave mESCs in trypsin for too long as it will harm the cells. 
Please cite this article as: Witteveldt and Macias, (2019). Differentiation of Mouse Embryonic Stem Cells to Neuronal Cells Using Hanging Droplets and

4. Transfer the required number of cells to a new flask with the appropriate amount of mESC medium containing LIF. Trypsin can be removed by centrifugation, but this is not necessary for v6.5 cells.

Note: Take care when pipetting mESCs with small (non-serological) pipette tips, they can be easily damaged.

5. Split mESCs every 2-3 days, avoid letting mESCs getting over-confluent.

Note: Avoid culturing mESCs for more than 10 passages.

D. Preparing embryoid bodies (EBs) using hanging droplets (Day 1, see also Video 1 for Steps B5-B7)

1. Trypsinize $\mathrm{mESCs}$ as described above, be sure to have single cells.

2. Resuspend in mESC medium without LIF.

3. Carefully count the cells using a hemocytometer or any other preferred method.

4. Adjust concentration to approximately $2 \times 10^{4}$ cells $/ \mathrm{ml}$ using $\mathrm{mESC}$ medium without LIF. Note: Slight deviations from this concentration is not a problem.

5. Using a multichannel pipette, place $20 \mu \mathrm{l}$ droplets of the cell suspension on the inside of a plastic Petri dish lid (not tissue-culture treated and not gelatin-coated). We aim for approximately 100 droplets on a $90 \mathrm{~mm}$ plate but depending on how many EBs are required, the size of the dish can be easily scaled up or down.

Note: Avoid touching the surface of the Petri dish with the pipette tip as this tends to make the droplets flatter and more spread out.

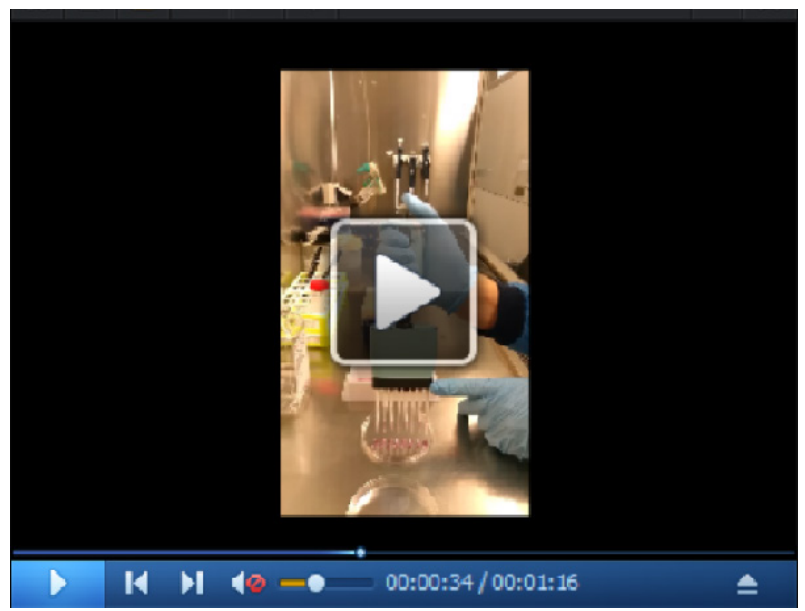

\section{Video 1. Preparation of hanging droplets on Petri dish}

6. Add $10 \mathrm{ml}$ of water or PBS to the bottom half of the Petri dish to prevent desiccation.

7. In one smooth movement, turn the lid with the droplets upside down and place on top of the bottom part of the Petri dish containing water or PBS.

8. Place in $37^{\circ} \mathrm{C}, 5 \% \mathrm{CO}_{2}$ incubator for $48 \mathrm{~h}$ (Day 3).

9. EB formation can be observed directly using a non-inverted microscope or using an inverted microscope by placing the lid with hanging droplets onto the empty bottom part of a Petri dish 
followed by carefully inverting the Petri dish. EBs should form within $24 \mathrm{~h}$. See Figure 2 for example of EBs of $v 6.5$ cells $24 \mathrm{~h}$ after preparation of hanging droplets.

Note: Differentiation can be confirmed by measuring the downregulation of pluripotency markers such as Nanog, Pou5f1 and Sox2 by quantitative real time PCR or Western blot.
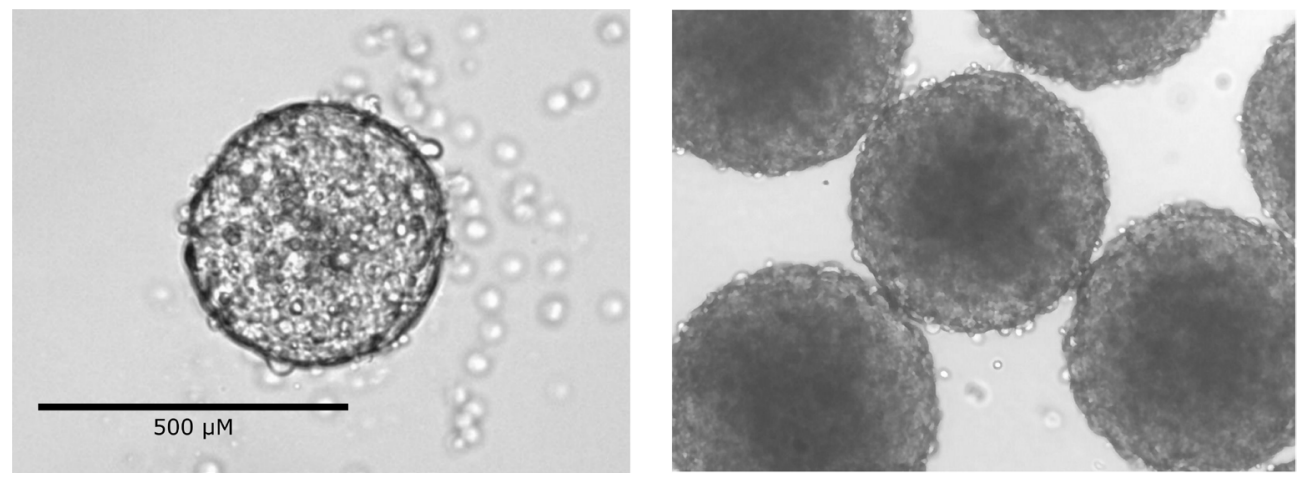

Figure 2. Typical morphology of embryoid body. v6.5 cells were cultured in hanging droplets in mESC medium without LIF for $24 \mathrm{~h}$ (left) and $48 \mathrm{~h}$ (right).

E. Differentiation of EBs using retinoic acid

1. After $48 \mathrm{~h}$ in droplets, the EBs are collected by adding PBS to the Petri dish lids and collecting the supernatant containing the EBs.

Note: It might require some pipetting up and down to dislodge all the EBs as they tend to stick to the plastic surface. Use serological pipettes as smaller pipettes are likely to damage the EBs.

2. The supernatant containing EBs can be collected in either 15 or $50 \mathrm{ml}$ tubes depending on the volume used.

3. Leave the tubes upright for $5 \mathrm{~min}$ or more to allow the EBs to settle on the bottom of the tubes. Remove the supernatant and resuspend the EBs in $10 \mathrm{ml}$ mESC medium without LIF.

4. Add the $10 \mathrm{ml}$ EB suspension to a $90 \mathrm{~mm}$ Petri dish (not culture treated and not gelatin-coated) and grow for another $48 \mathrm{~h}$ at $37^{\circ} \mathrm{C}$ and $5 \% \mathrm{CO}_{2}$ (day 5).

Note: The resuspension volume and size of the plate can be adjusted to the number of EBs required, we aim for approximately $100 \mathrm{EBs} / 90 \mathrm{~mm}$ dish.

5. After $48 \mathrm{~h}$ of incubation, replace the medium with $10 \mathrm{ml}$ of fresh mESC medium without LIF using the method described in Steps E2 and E3 and add Retinoic acid to a final concentration of $250 \mathrm{nM}$.

Note: The concentration of Retinoic acid is likely to be cell line dependent.

6. Continue to culture for another 7 days (Day 12) whilst replacing the medium with fresh $\mathrm{mESC}$ medium without LIF supplemented with $250 \mathrm{nM}$ retinoic acid every 2 days. See Figure 3 for example of EB 7 days after RA treatment. 




Figure 3. Embryoid bodies after 7 days of retinoic acid treatment. Scale bar $=500 \mu \mathrm{m}$.

7. After these 7 days (on Day 12), the EBs can be collected as described in Steps D2 and D3 and seeded on tissue-cultured treated, gelatin-coated plates or flasks. At this point, EBs will attach and cells will start to migrate from the EBs within $24 \mathrm{~h}$. See Figure 4 for example of EB $24 \mathrm{~h}$ after attachment to gelatin-coated plate and Figure 5 for EBs 7 days after attachment, showing considerable migration and growth of cells of neuronal lineage.

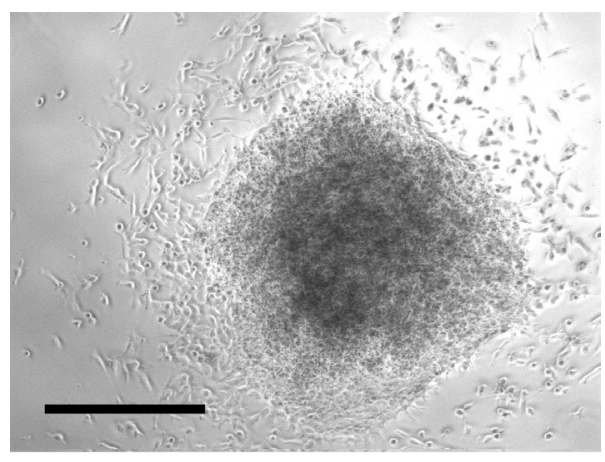

Figure 4. Embryoid bodies $24 \mathrm{~h}$ after plating on gelatin-coated plates showing migration of differentiated cells from embryoid body. Scale bar $=500 \mu \mathrm{m}$.

8. At this point, the cells are differentiated into cells of neuronal lineage and can be directly used for further experiments or frozen down.

Note: Differentiation into cell of neuronal lineage can be verified through measuring gene expression of genes such as Neurog2, Gata4, Gata6, Nestin, Sox1 and TuJ1 by quantitative real time PCR or Western blot (Ying et al., 2003; Witteveldt et al., 2019). To ensure a homogeneously differentiated population, lineage selection by cell-sorting based on fluorophore-expression driven by lineage-specific promoters or immune-isolation by lineagespecific extracellular markers (Jüngling et al., 2003; Ladewig et al., 2008) might be necessary. When multiple replicates are required we suggest to differentiate individual replicates. 

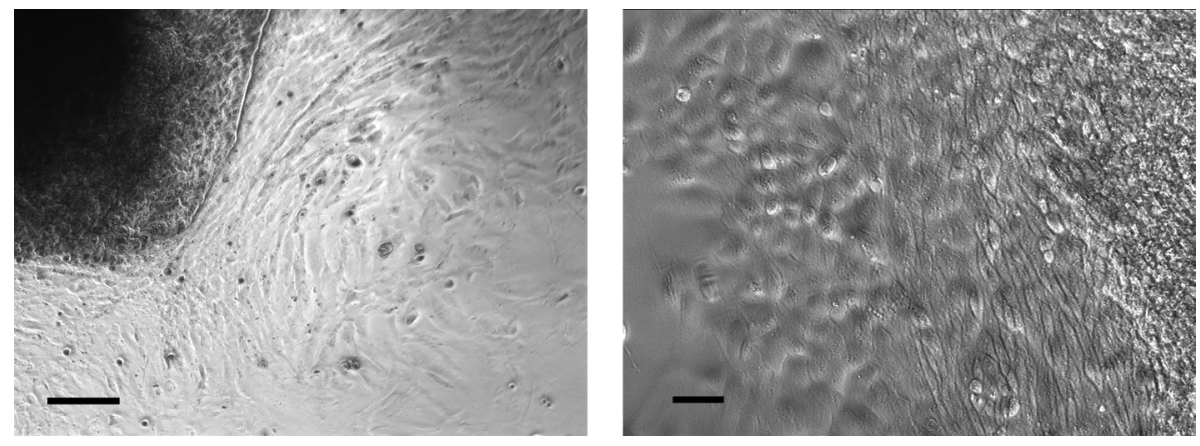

Figure 5. Embryoid bodies 7 days after plating on gelatin coated plates showing migration and growth of differentiated cells of neuronal lineage with clearly changed morphology. Scale bar $=20 \mu \mathrm{m}$.

\section{$\underline{\text { Recipes }}$}

1. mESC medium with LIF

$415 \mathrm{ml}$ DMEM

$5 \mathrm{ml}$ Penicillin/Streptomycin

$5 \mathrm{ml}$ MEM Non-Essential amino acids

$400 \mu \mathrm{l}$ 2-Mercaptoethanol (40 $\mu \mathrm{M}$ final concentration)

$75 \mathrm{ml}$ Fetal bovine serum (15\% final concentration)

$50 \mu \mathrm{LIF}$ (final concentration of $1000 \mathrm{U} / \mathrm{ml}$ )

2. mESC medium without LIF

$415 \mathrm{ml}$ DMEM

$5 \mathrm{ml}$ Penicillin/Streptomycin

$5 \mathrm{ml}$ MEM Non-Essential amino acids

$400 \mu \mathrm{l}$ 2-Mercaptoethanol (40 $\mu \mathrm{M}$ final concentration)

$75 \mathrm{ml}$ Fetal bovine serum (15\% final concentration)

3. $0.05 \%$ Trypsin

$10 \mathrm{ml}$ of $0.25 \%$ Trypsin (stock)

$40 \mathrm{ml}$ of PBS

\section{Acknowledgments}

This protocol was originally published in a shorter form in Witteveldt et al. (2019). Its development and implementation were financially supported by the Wellcome Trust (107665/Z/15/Z).

\section{Competing interests}

The authors declare no conflict of interests. 


\section{References}

1. Abranches, E., Silva, M., Pradier, L., Schulz, H., Hummel, O., Henrique, D. and Bekman E. (2009). Neural differentiation of embryonic stem cells in vitro: a road map to neurogenesis in the embryo. PLoS One 4(7): e6286

2. Itskovitz-Eldor, J., Schuldiner, M., Karsenti, D., Eden, A., Yanuka, O., Amit, M., Soreq, H. and Benvenisty, N. (2000). Differentiation of human embryonic stem cells into embryoid bodies compromising the three embryonic germ layers. Mol Med 6(2): 88-95.

3. Jones-Villeneuve, E. M., Rudnicki, M. A., Harris, J. F. and McBurney, M. W. (1983). Retinoic acid-induced neural differentiation of embryonal carcinoma cells. Mol Cell Biol 3(12): 2271-2279.

4. Witteveldt, J., Knol, L. I. and Macias, S. (2019). MicroRNA-deficient mouse embryonic stem cells acquire a functional interferon response. Elife 8: e44171.

5. Wobus, A. M., Wallukat, G. and Hescheler, J. (1991). Pluripotent mouse embryonic stem cells are able to differentiate into cardiomyocytes expressing chronotropic responses to adrenergic and cholinergic agents and $\mathrm{Ca}^{2+}$ channel blockers. Differentiation 48(3): 173-182.

6. Ying, Q. L., Stavridis, M., Griffiths, D., Li, M. and Smith, A. (2003). Conversion of embryonic stem cells into neuroectodermal precursors in adherent monoculture. Nat Biotechnol 21(2): 183186.

7. Ladewig, J., Koch, P., Endl, E., Meiners, B., Opitz, T., Couillard-Despres, S., Aigner, L. and Brüstle, O. (2008). Lineage selection of functional and cryopreservable human embryonic stem cell-derived neurons. Stem Cells 26(7):1705-1712.

8. Jüngling, K., Nägler, K., Pfrieger, F. W. and Gottmann, K. (2003). Purification of embryonic stem cell-derived neurons by immunoisolation. FASEB J 17(14):2100-2. 